\title{
Solitary Multilocular Kidney Cyst
}

National Cancer Institute

\section{Source}

National Cancer Institute. Solitary Multilocular Kidney Cyst. NCI Thesaurus. Code C103919.

A single cyst in the kidney with several compartments. 